University of Nebraska - Lincoln

DigitalCommons@University of Nebraska - Lincoln

Faculty Publications from the Center for Plant

Science Innovation

2015

Redirection of metabolic flux for high levels of omega-7

monounsaturated fatty acid accumulation in camelina seeds

Huu Tam Nguyen

Hyunwoo Park

Karen L. Koster

Rebecca E. Cahoon

Hanh Nguyen

See next page for additional authors

Follow this and additional works at: https://digitalcommons.unl.edu/plantscifacpub

Part of the Plant Biology Commons, Plant Breeding and Genetics Commons, and the Plant Pathology

Commons

This Article is brought to you for free and open access by the Plant Science Innovation, Center for at DigitalCommons@University of Nebraska - Lincoln. It has been accepted for inclusion in Faculty Publications from the Center for Plant Science Innovation by an authorized administrator of DigitalCommons@University of Nebraska - Lincoln. 


\section{Authors}

Huu Tam Nguyen, Hyunwoo Park, Karen L. Koster, Rebecca E. Cahoon, Hanh Nguyen, John Shanklin, Thomas E. Clemente, and Edgar B. Cahoon 


\title{
Redirection of metabolic flux for high levels of omega-7 monounsaturated fatty acid accumulation in camelina seeds
}

\author{
Huu Tam Nguyen ${ }^{1}$, Hyunwoo Park ${ }^{2}$, Karen L. Koster ${ }^{3}$, Rebecca E. Cahoon ${ }^{1}$, Hanh T. M. Nguyen², John Shanklin ${ }^{4}$, \\ Thomas E. Clemente ${ }^{2}$ and Edgar B. Cahoon ${ }^{1, *}$ \\ ${ }^{1}$ Center for Plant Science Innovation and Department of Biochemistry, University of Nebraska-Lincoln, Lincoln, NE, USA \\ ${ }^{2}$ Center for Plant Science Innovation and Department of Agronomy \& Horticulture, University of Nebraska-Lincoln, Lincoln, NE, USA \\ ${ }^{3}$ Department of Biology, The University of South Dakota, Vermillion, SD, USA \\ ${ }^{4}$ Biosciences Department, Brookhaven National Laboratory, Upton, NY, USA
}

Received 28 April 2014;

revised 29 June 2014;

accepted 30 June 2014.

*Correspondence (Tel +1 402472 5611;

fax +1 402472 3139;

email ecahoon2@unl.edu)

Keywords: omega-7 fatty acid, monounsaturated fatty acid, oilseed, metabolic engineering, fatty acid, camelina.

\begin{abstract}
Summary
Seed oils enriched in omega-7 monounsaturated fatty acids, including palmitoleic acid (16:1 $\Delta 9$ ) and cis-vaccenic acid (18:1 $\Delta 11)$, have nutraceutical and industrial value for polyethylene production and biofuels. Existing oilseed crops accumulate only small amounts $(<2 \%)$ of these novel fatty acids in their seed oils. We demonstrate a strategy for enhanced production of omega-7 monounsaturated fatty acids in camelina (Camelina sativa) and soybean (Glycine max) that is dependent on redirection of metabolic flux from the typical $\Delta 9$ desaturation of stearoyl (18:0)-acyl carrier protein $(A C P)$ to $\Delta 9$ desaturation of palmitoyl (16:0)-acyl carrier protein (ACP) and coenzyme $A(C O A)$. This was achieved by seed-specific co-expression of a mutant $\triangle 9$-acyl-ACP and an acyl-CoA desaturase with high specificity for 16:0-ACP and CoA substrates, respectively. This strategy was most effective in camelina where seed oils with $\sim 17 \%$ omega- 7 monounsaturated fatty acids were obtained. Further increases in omega- 7 fatty acid accumulation to $60-65 \%$ of the total fatty acids in camelina seeds were achieved by inclusion of seed-specific suppression of 3-keto-acyl-ACP synthase ll and the FatB 16:0-ACP thioesterase genes to increase substrate pool sizes of 16:0-ACP for the $\triangle 9$-acyl-ACP desaturase and by blocking C 18 fatty acid elongation. Seeds from these lines also had total saturated fatty acids reduced to $\sim 5 \%$ of the seed oil versus $12 \%$ in seeds of nontransformed plants. Consistent with accumulation of triacylglycerol species with shorter fatty acid chain lengths and increased monounsaturation, seed oils from engineered lines had marked shifts in thermotropic properties that may be of value for biofuel applications.
\end{abstract}

\section{Introduction}

Oils containing omega-7 unsaturated fatty acids (FA), comprising primarily palmitoleic acid $(16: 1 \Delta 9)$ and its elongation product cisvaccenic acid (18:1 111 ; Figure $S 1)$, are not typically abundant in plant seeds but are enriched in seeds of several nonagronomic plant species, including seeds of cat's claw creeper (Dolichandra unguis-cati or Doxantha unguis-cati) that accumulate up to $64 \%$

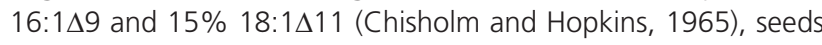
of sea buckthorn (Hippophae rhamnoides) that accumulate $32 \%$ 16:1 19 (Kallio et al., 2002), and seeds of macadamia (Macadamia sp.) that accumulate $20 \%$ to $30 \%$ 16:1 19 (Bridge and Hilditch, 1950; Saleeb et al., 1973). These fatty acids have uses for a number of industrial applications, ranging from polymer precursor production and biodiesel formulations, and for nutraceuticals. Biodiesel containing fatty acid methyl esters produced from vegetable oils with high monounsaturated fatty acid content, including those with high omega-7 monounsaturated fatty acid content, has superior functional properties relative to biodiesel from common vegetable oils, such as soybean oil, with high polyunsaturated fatty acid levels (Ciubota-Rosie et al., 2013; Durrett et al., 2008; Knothe et al., 2005). These include enhanced oxidative stability, $\mathrm{NO}_{x}$ emission, and ignition quality conferred by increased fatty acid monounsaturation and reduced polyunsaturation (Durrett et al., 2008). In addition, the melting point of the omega- 7 fatty acid palmitoleic acid is $\sim 15{ }^{\circ} \mathrm{C}$ lower than that of the omega- 9 fatty acid oleic acid, resulting in better cold flow properties for palmitoleic acid-rich oils relative to oleic acid-rich oils. Omega-7 fatty acids also have considerable potential as a feedstock for the production of 1-octene using metathesis chemistry (del Cardayre, 2013; Meier, 2009; Rybak et al., 2008). 1-Octene is used commercially as a monomer in the production of polyethylene, including low linear density polyethylene (LLDPE). 1-Octene is currently derived from petroleum. As such, vegetable oils rich in omega-7 fatty acid acids can serve as renewable sources of this industrially important compound. Vegetable oils enriched in omega-7 fatty acids have also been ascribed a number of beneficial health properties, including promotion of cardiovascular fitness and reduction in LDL cholesterol levels (Curb et al., 2000; Mozaffarian et al., 2010; Yang et al., 2011). Currently, the major nutraceutical source of omega7 fatty acid oils is sea buckthorn seeds (Kallio et al., 2002).

No commercially significant oilseed crops currently produce high levels of omega-7 fatty acid-rich oils. Common vegetable oils, such as soybean oil, typically contain $<2 \%$ of omega- 7 monounsaturated fatty acids, whereas plants such as cat's claw creeper that accumulate high levels of of omega-7 fatty acids in their seed oils have little agronomic potential. As a result, metabolic engineering of omega-7 fatty acid production in existing oilseeds is the most promising approach for generating 
vegetable oils enriched in these fatty acids. The initial challenge for engineering omega-7 fatty acid production is that unsaturated fatty acids in oilseeds are formed principally through a series of desaturation reactions beginning with $\Delta 9$ desaturation of stearoyl (18:0)-acyl carrier protein (ACP) by the plastidlocalized $\triangle 9-18: 0-A C P$ desaturase. This reaction gives rise to the $\omega-9$ unsaturated oleic acid fatty acid. One approach for generating the omega-7 unsaturated palmitoleic acid is $\Delta 9$ desaturation of palmitic acid as an ACP- or coenzyme A (COA)linked substrate (Figures 1 and S1). The feasibility of this approach was shown by seed-specific expression in Arabidopsis of a mutated $\triangle 9-18: 0-A C P$ desaturase with enhanced substrate specificity for 16:0-ACP (Cahoon and Shanklin, 2000). Using a 3-ketoacyl-ACP synthase II (KASII) mutant with elevated 16:0ACP substrate pools as a background, Arabidopsis seeds were obtained with $\sim 30 \%$ omega-7 fatty acids, including 16:1 19 and its elongation products $18: 1 \Delta 11$ and 20:1 113 (Cahoon and Shanklin, 2000). Levels of $67 \%$ of $16: 1 \Delta 9$ and $18: 1 \Delta 11$ were subsequently achieved in Arabidopsis seeds by inclusion of the seed-specific expression of fungal 16:0-/18:0-COA desaturases (targeted to the cytosol) and RNAi silencing of $F A E 1$, encoding the 3-ketoacyl-COA synthase responsible for the elongation of C18 and C20 fatty acids (Nguyen et al., 2010).

In this report, we have explored the efficacy of this strategy primarily in camelina (Camelina sativa), an emerging Brassicaceae oilseed crop that is particularly suitable for the complex metabolic engineering required for the introduction of the omega-7 fatty acid biosynthetic pathway. Camelina is amenable to Agrobacterium-based transformation using a floral vacuum infiltration method (Lu and Kang, 2008). We have previously developed an extensive metabolic engineering tool box of seed-specific pro- moters and selection markers to facilitate these studies (Horn et al., 2013; Nguyen et al., 2013). Here, we have systematically examined the use of a six transgene strategy incorporating the previously described method from Arabidopsis (Cahoon and Shanklin, 2000; Nguyen et al., 2010) that also combined RNAi silencing of FatB, encoding the 16:0-ACP thioesterase. Through this approach, camelina oil was generated with $\sim 66 \%$ omega-7 fatty acids as well as an unexpected two- to threefold reduction in total saturated fatty acid content relative to conventional camelina oil. These compositional changes were found to significantly impact of the thermotropic properties of camelina oil. We also report the evaluation of a partial metabolic engineering strategy to confer omega-7 fatty acid accumulation in soybean.

\section{Results}

Metabolic engineering for enhanced accumulation of omega-7 unsaturated fatty acids in camelina seeds

Experiments were undertaken to increase the omega-7 unsaturated fatty acid content of camelina seeds by systematically testing transgenes and transgene combinations predicted to enhance flux towards the synthesis of palmitoleic (16:1 $1 \Delta 9$ or omega-7) and cis-vaccenic acids (18:1 111 or omega-7), which account for $<2 \%$ of the fatty acids of wild-type camelina seeds. For these experiments, seeds were evaluated from 5 to 10 independent $T_{1}$ lines for each construct. The initial goal was to remodel plastidic pathways by shifting monounsaturated fatty acid synthesis to the $\Delta 9$ or omega- 7 desaturation of palmitoyl (16:0)-ACP rather than the $\Delta 9$ desaturation of stearoyl (18:0)ACP that typically generates $\sim 90 \%$ of the fatty acids released from camelina seed plastids (Figure 1). To enhance substrate

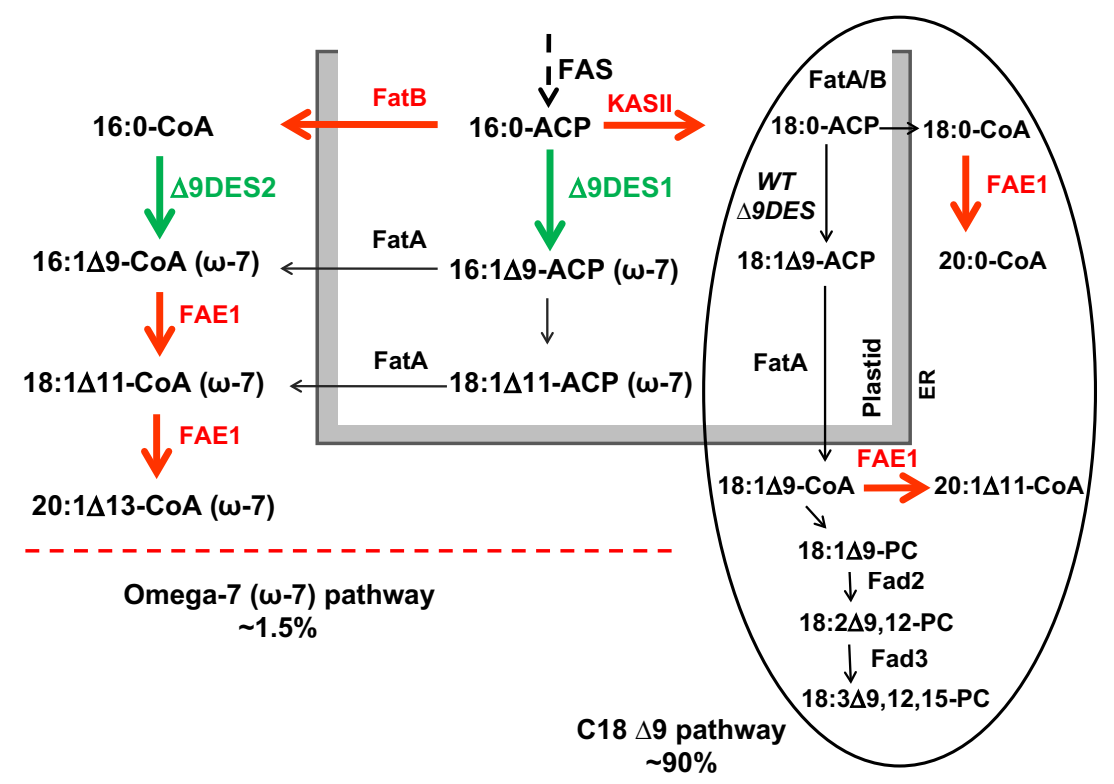

Figure 1 Schematic of the metabolic engineering strategy for enhancement of omega-7 monounsaturated fatty acid biosynthesis. Arrows shown in green indicate up-regulated metabolic steps and red arrows indicate down-regulated metabolic steps. The metabolic engineering strategy involves introduction and up-regulation of a plastid-localized mutant $\triangle 9$ acyl-ACP desaturase ( $\triangle 9 D E S 1$; Com25) with enhanced 16:0-ACP activity and an ER-localized $\triangle 9$ 16:0-CoAspecific desaturase ( $\triangle 9 D E S 2)$. RNAi suppression of genes for the plastid-localized 3-ketoacyl-ACP synthase II (KASII) and the FatB 16:0-ACP thioesterase (FatB) increases pool sizes of 16:0-ACP for the $\triangle 9-16: 0-A C P$ desaturase ( $\triangle 9 D E S 1)$. RNAi suppression of the FATTY ACID ELONGATION 1 (FAE1) gene reduces elongation of primarily C18 fatty acids to C20 moieties. As shown, the omega-7 desaturation pathway yields $<1.5 \%$ omega-7 unsaturated fatty acids in nonengineered camelina seeds, whereas the typical C18 $\Delta 9$ pathway typically yields $\sim 90 \%$ of the fatty acids in nonengineered camelina seeds. 
pool sizes of $16: 0-A C P$ for $\Delta 9$ desaturation, $\beta$-ketoacyl-ACP synthase II (KASII) genes were suppressed by RNAi ('HP'; Figure 2a). The expected outcome was reduced 16:0- to 18:0ACP elongation. Consistent with this, seed-specific expression of a camelina KASII RNAi (KASII-HP) transgene yielded an increase in palmitic acid content from $7 \%$ in wild-type seeds to $31 \%$ in DsRed-positive seeds from $T_{1}$ seeds of 10 independent lines (Table 1). This was also accompanied by an increase in total omega-7 unsaturated fatty acids, principally as 16:1 $\Delta 9$, from $1.4 \%$ in wild-type seeds to $9 \%$ in the KASII-HP seeds (Table 1 and Figure $3 a, b)$. The mutated $\triangle 9$-acyl-ACP desaturase Com25 was then expressed alone or in combination with the $C$. elegans Fat5 ER $\triangle 9$-16:0-CoA desaturase (Fahy et al., 2013; Watts and Browse, 2000) in camelina seeds. The result was an increase in omega-7 unsaturated fatty acid content in DsRed-positive seeds from $\mathrm{T}_{1}$ plants of independent events expressing Com 25 to $17 \%$ of the total fatty acids, including 2\% 16:1 $\Delta 9,8 \% 18: 1 \Delta 11$ and $7 \% 20: 1 \Delta 13$ (Table 1). The combination of seed-specific expression of Com25 and Fat5 resulted in additional increases in omega-7 unsaturated fatty acids to $23 \%$ of the total fatty acids, including $2.7 \% \quad 16: 1 \Delta 9,11 \% \quad 18: 1 \Delta 11$ and $9 \% \quad 20: 1 \Delta 13$ (Table 1 and Figure 3c). Of note, these lines were not advanced beyond the $T_{1}$ generation and genetic complexity of transgenes was not assessed. As such, it cannot be excluded that multiple transgene insertions may affect the total amount of omega-7 fatty acids accumulated in the engineered seeds.
Combining Com25 and Fat5 expression with KASII-HP increased omega-7 unsaturated fatty acids in seeds from homozygous $\mathrm{T}_{3}$ lines to as high as $43 \%$ of the total fatty acids, including $16.5 \% \quad 16: 1 \Delta 9, \quad 11 \% \quad 18: 1 \Delta 11$ and $13 \% \quad 20: 1 \Delta 13$ (Table 1 and Figure $3 \mathrm{~d}$ ). To reduce the production of C20 omega-7 unsaturated fatty acids, seed-specific silencing of FAE1 (FAE-HP) was introduced with Com25, Fat5 and KASII-HP. The result was a small increase in total omega- 7 unsaturated fatty acids in camelina seeds from homozygous $T_{3}$ lines to as high as $44 \%$ of the total fatty acids, but a large increase in 16:1 $\Delta 9$ and $18: 1 \Delta 11$ to $24 \%$ and $18 \%$ of the total fatty acids and a reduction in $20: 1 \Delta 13$ to $<1 \%$ of the total fatty acids (Table 1 , Figure 3e).

To further increase total omega-7 unsaturated fatty acids, a second copy of the Com25 transgene was introduced along with RNAi suppression of the FatB 16:0-ACP thioesterase (FatB-HP; Figure 2b) into the Com25, Fat5, KASII-HP, FAE1-HP line. The total omega-7 unsaturated fatty acid content of the resulting engineered seeds from homozygous $T_{3}$ lines was as high as $65 \%$ of the total fatty acids, including $\sim 3 \%$ of $16: 2$ and $16: 3$ derived from 16:1 19 in the highest omega-7 producing line (Table 1, Figure 3f, Figure S2). The primary omega-7 monounsaturated fatty acids of the engineered seeds were $16: 1 \Delta 9$ (32\% of total fatty acids) and 18:1 111 (30\% of the total fatty acids) (Table 1 and Figure $4 a$ ). Of note, although the glycinin-1 seed-specific promoter was used in five of the transgenes, the stability of the

(a)

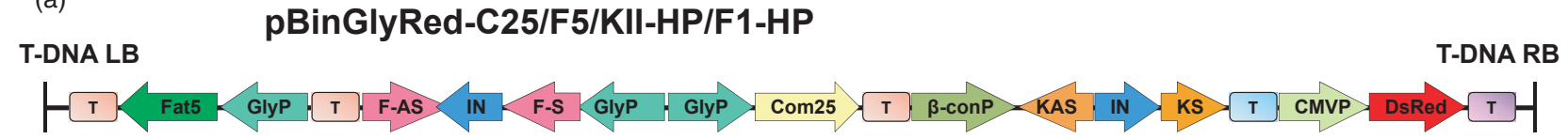

(b) T-DNA LB pBinGlyBAR1-C25/FatB-HP

T-DNA RB
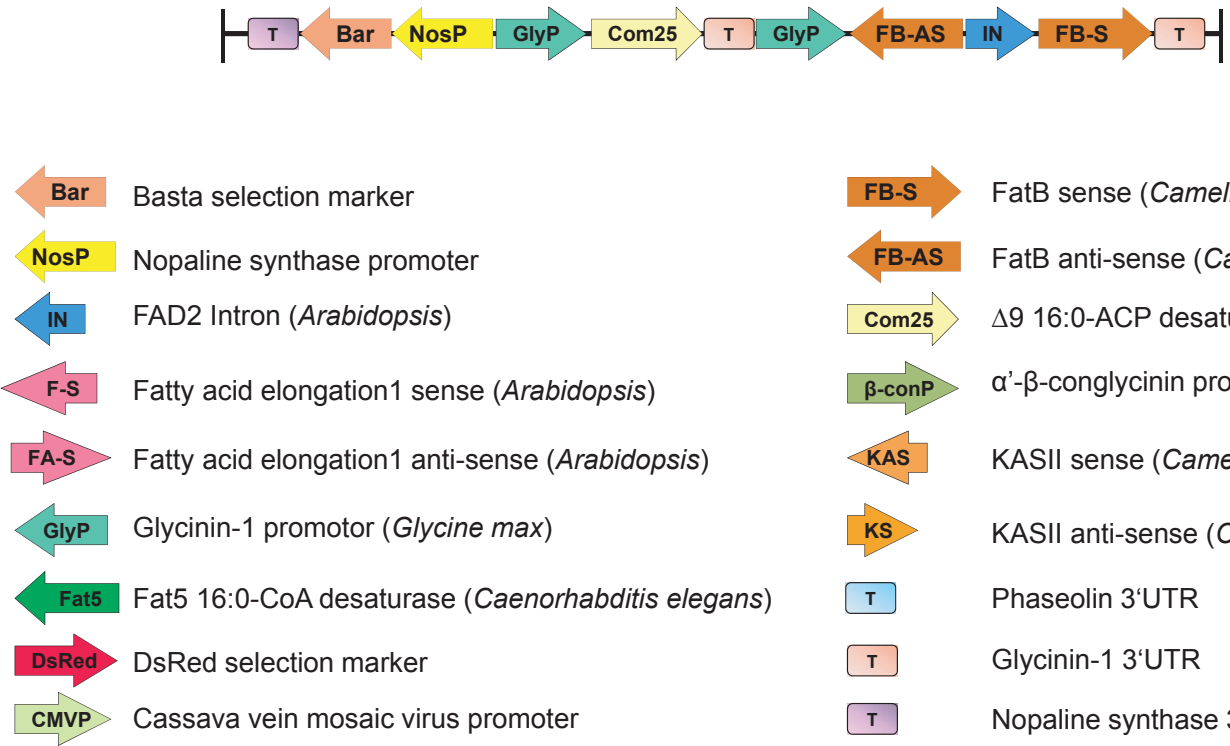

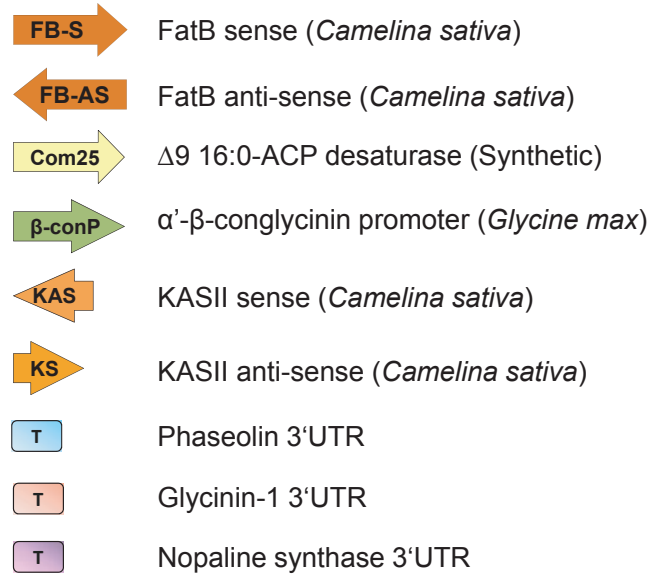

Figure 2 T-DNAs used for generating high omega-7 unsaturated fatty acids in camelina seeds. As shown in (a), pBinGlyRed-C25/F5/KII-HP/F1-HP contains seed-specific cassettes for expression of the $\triangle 9$-16:0-ACP desaturase (Com25) and the $\triangle 9-16: 0-C o A$ desaturase genes (Fat5) and RNAi suppression of genes for 3-ketoacyl-ACP synthase (KASII) and fatty acid-CoA elongase (FAE1) with sense and antisense hairpin arms for the target genes. Selection is accomplished with a DsRed fluorescence marker. As shown in (b), a second T-DNA pBinGlyBAR1-C25/FatB-HP was used for retransformation of camelina that contains seed-specific cassettes for the expression of Com25 and for RNAi suppression of the FatB 16:0-ACP thioesterase. Selection is achieved by use of a Basta resistance cassette. Constitutive and seed-specific promoters, $3^{\prime}$ UTR sequences, and arrangements of cassettes are also shown. 


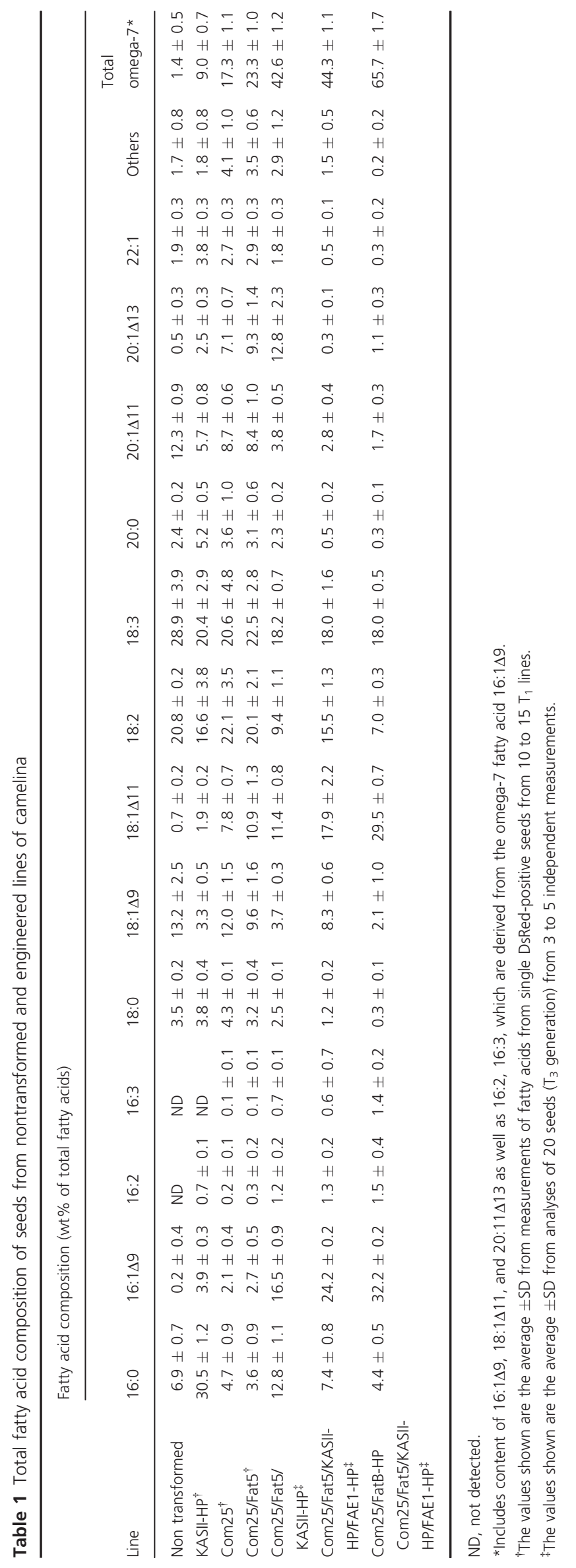


(a)

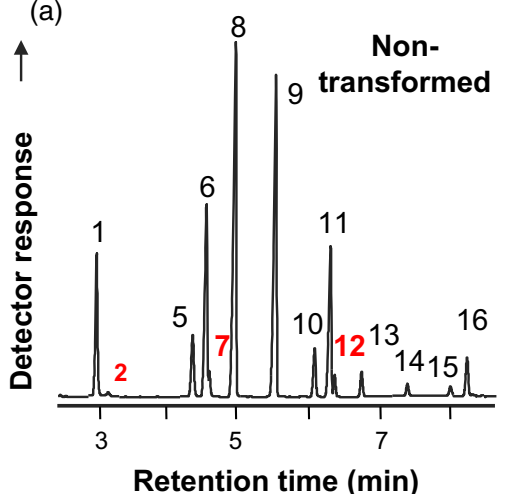

(b)

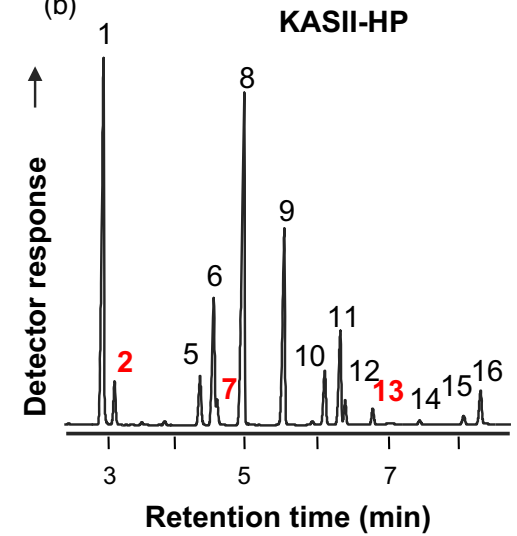

(c)

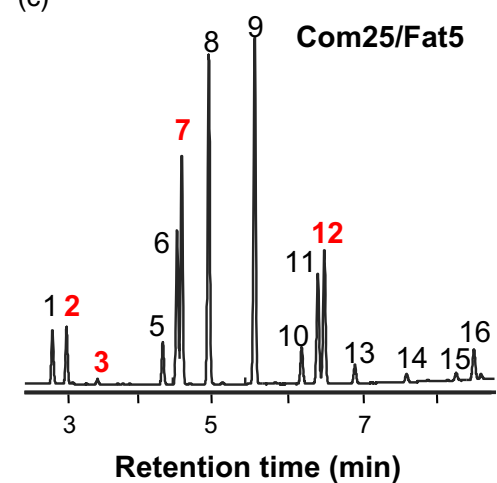

(d)

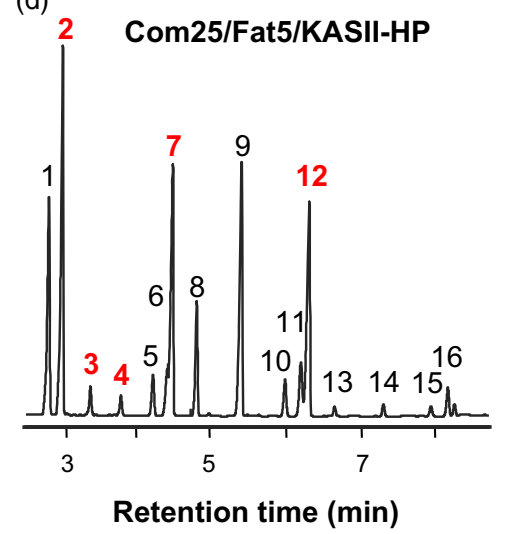

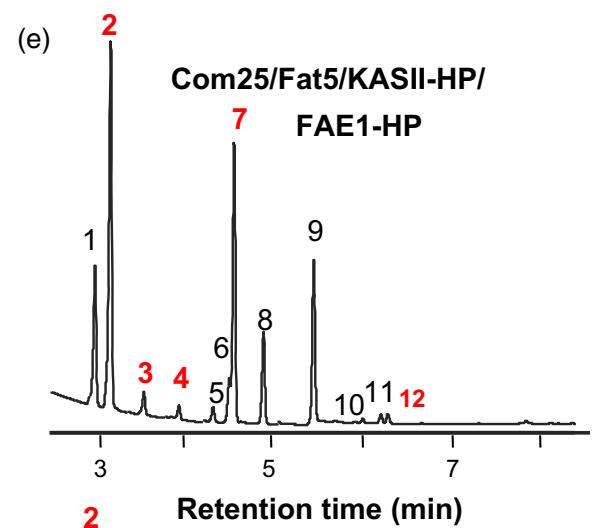

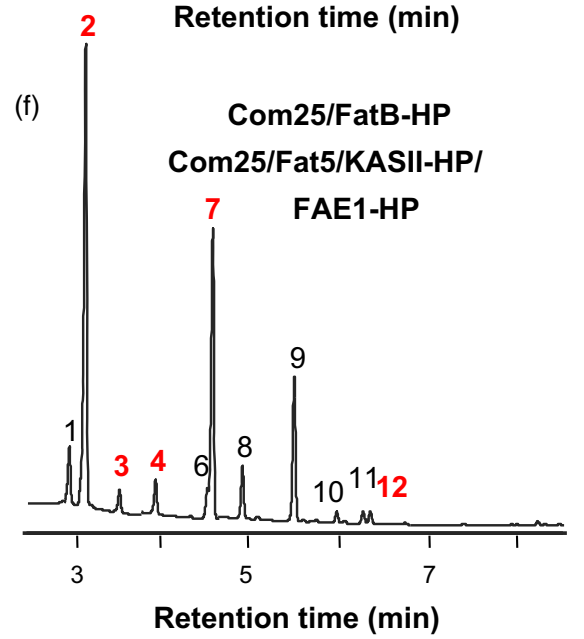

Figure 3 Representative gas chromatograms showing separation and relative quantification of fatty acid methyl esters generated from camelina seeds of nontransformed plants (a) and plants engineered for seed-specific expression of genes for $\Delta 9-16: 0$-ACP desaturase (Com25) and/or $\triangle 9$-16:0-CoA desaturase (Fat5) and/or RNAi suppression (indicated by 'HP') of genes for 3-ketoacyl-ACP synthase II (KASII), fatty acid elongation-1 (FAE1), and/or FatB 16:0-ACP thioesterase. The gas chromatograms in B-F are from seeds expressing a KASII-HP RNAi cassette (b), Com25 and Fat5 (c), Com25, Fat5, KASII-HP RNAi cassette (d), Com25, Fat5, KASII-HP and FAE1-HP RNAi cassettes (e), and Com25 and FatB-HP RNAi cassette + Com25, Fat5, and KASII-HP and FAE1-

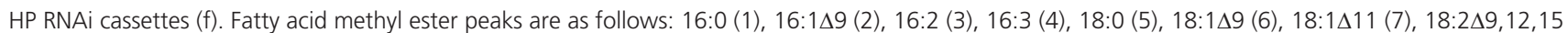

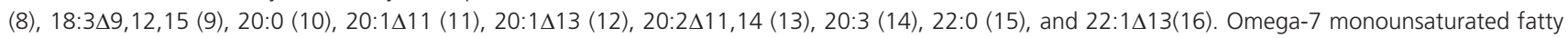
acids or omega-7 pathway-derived unsaturated fatty acids are shown with red labels.

omega-7 trait was observed over at least four generations. Double-bond positions of these fatty acids were confirmed to be in the omega-7 position by gas chromatography/mass spectrometry analysis of thiomethyl derivatives of fatty acid methyl esters (Figure S3). Another notable effect of these engineering efforts was a substantial decrease in the total saturated fatty acids. In this regard, the saturated fatty acid content of wild-type camelina seeds is $\sim 12 \%$ of total fatty acids, whereas seeds engineered with the six transgene combination had $\sim 5 \%$ saturated fatty acids, including reduction of palmitic acid content from $7 \%$ in nontransformed seeds to $4.4 \%$ in the engineered seeds and reduction of stearic acid from $3.5 \%$ in nontransformed seeds to $0.3 \%$ in the engineered seeds (Table 1 and Figure 4b).

Metabolic engineering for enhanced accumulation of omega-7 unsaturated fatty acids in soybean seeds

We also explored whether this engineering strategy can be used for enhanced omega-7 unsaturated fatty acid production in soybean seeds. The more labour- and skill-intensive transformation protocol for soybean limited the numbers of constructs that could be evaluated relative to camelina. In these experiments, the same transgene cassettes as used for camelina transformation were tested for seed-specific co-expression of Com25 and Fat5 and Com25 and Fat5 combined with KASII-HP in soybean. Nine to twelve independent transgenic events were generated for each construct. The top-performing line for Com25 and Fat5 expression resulted in the accumulation of omega- 7 unsaturated fatty acids to $\sim 4.5 \%$ of the total fatty acids, and the topperforming line for Com25 and Fat5 expression with KASII-HP resulted in the accumulation of omega-7 fatty acids to $~ 16.5 \%$ of the total fatty acids (Table 2, Figure S2). In both cases, the major omega-7 fatty acid that accumulated was 18:1 111 . Omega-7 unsaturated fatty acids were not detected in soybean seeds from nontransformed plants. Amounts of omega-7 monounsaturated fatty acids accumulated with both constructs were considerably lower than that achieved in camelina seeds with the same transgene cassettes.

Omega-7 unsaturated fatty acids are not excluded from phosphatidylcholine

The relative amounts of omega-7 unsaturated fatty acids were examined in triacylglycerol (TAG) and phosphatidylcholine (PC) of nontransformed camelina seeds and seeds engineered for 
(a)

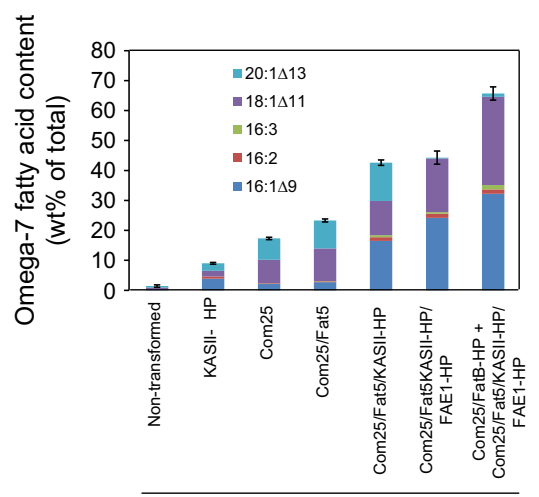

Camelina lines (b)

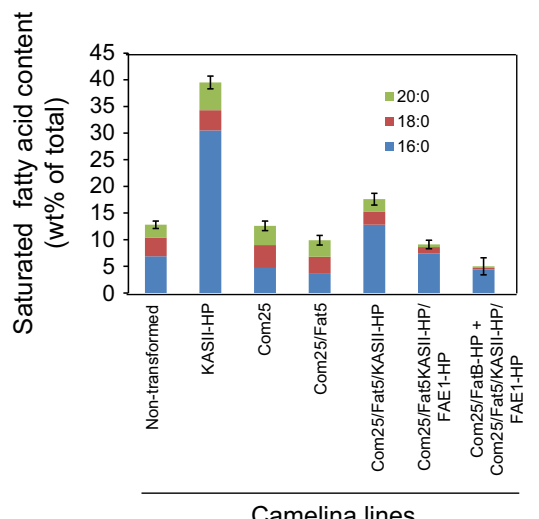

Figure 4 Comparison of amounts of omega-7 unsaturated fatty acids (a) and saturated fatty acids (b) in seeds of nontransformed plants or plants engineered for expression of 3-ketoacyl-ACP synthase II RNAi suppression cassette (KASII-HP); $\triangle 9$-16:0-ACP desaturase (Com25); Com25/A9-16:0-CoA desaturase (Fat5); Com25/Fat5/KASII-HP; Com25/Fat5/KASII-HP/fatty acid elongation1 RNAi suppression cassette (FAE1-HP); and Com25/FatB RNAi suppression cassette (FatB-HP) + Com25/Fat5/KASII-HP/FAE1-HP. Values shown are the averages of total omega-7 unsaturated fatty acids (a) or total saturated fatty acids (b) in 10 to 15 single seeds from $T_{1}$ lines of highest omega-7 unsaturated fatty acid accumulators \pm SD for KASII-HP, Com25, and Com25/Fat5 constructs or 20 seeds from nontransformed lines or $T_{3}$ homozygous plants of highest omega-7 unsaturated fatty acid accumulating lines \pm SD ( $n=3-5)$ for Com25/Fat5/KASII-HP, Com25/Fat5/KASII-HP/FAE1-HP, Com25/FatB-HP + Com25/Fat5/KASII-HP/FAE1-HP constructs. Included in the total amounts of omega-7 unsaturated fatty acids are amounts of 16:2 and 16:3, which are derived from the omega-7 unsaturated fatty acid $16: 1 \Delta 9$.

highest omega-7 unsaturated fatty acid production (from plants transformed with six transgenes). In seed lipid extracts from high omega-7 unsaturated fatty acid-producing seeds, the content of $16: 1 \Delta 9$ and $18: 1 \Delta 11$ was remarkably similar in the PC and TAG fractions (Table 3 ). In each of these fractions, the $16: 1 \Delta 9$ content was $\sim 32 \%$ of the total fatty acids, and the $18: 1 \Delta 11$ content was $\sim 29-34 \%$ of the total fatty acids. By comparison, the omega-7 unsaturated fatty acid content of TAG and PC from nontransformed camelina seeds was $\leq 1.1$ of the fatty acids of these lipids (Table 3 ). These results suggest that camelina seeds do not exclude omega-7 fatty acids from membrane lipids.

\section{Omega-7 unsaturated fatty acid accumulation is accompanied by large changes in TAG molecular species}

Intact, ammoniated $\left(\mathrm{M}+\mathrm{NH}_{4}\right)$ TAG molecular species were profiled by ESI-MS/MS by direct infusion of neutral lipid extracts from nontransformed camelina seeds and seeds engineered for high omega-7 unsaturated fatty acid production ( $65 \%$ of the total fatty acids). Neutral loss scans were performed to validate the fatty acid components of the intact TAG molecular species. Product ion spectra of individual peaks were also obtained to further confirm fatty acid compositions of individual peaks and the number of different TAG species having the identical mass present within a given peak. While the positional distribution of the fatty acids can be suggested by the relative abundance of the two DAG fragments produced, positional distribution cannot be firmly established by this method. Wild-type camelina oil is rich in TAGs containing 52, 54 and 56 carbons, with 16:0/18:2/18:3 $(870.8 \mathrm{~m} / \mathrm{z})$, a mixture of tri-18:2 and 18:1/18:2/18:3 $(896.8 \mathrm{~m} / \mathrm{z})$, and $20: 1 / 18: 2 / 18: 3(924.8 \mathrm{~m} / \mathrm{z})$ representing the most abundant ammoniated $\left(\mathrm{M}+\mathrm{NH}_{4}\right)$ TAG species (Figure 5a). In contrast, high omega-7 camelina oil contains TAG species with lower total carbon numbers (48, 50 and 52 carbons). Neutral loss scanning and product ion spectra of individual peaks confirm the presence of $16: 1$ and 18:1 in the most abundant TAG molecular species (Figure 5b; Figures 54 and S5). For example, the product ion spectrum of the prominent peak at $818.8 \mathrm{~m} / \mathrm{z}$ confirms that this peak is composed entirely of $16: 1 / 16: 1 / 16: 1$ TAG. Similarly, the peak at $846.8 \mathrm{~m} / \mathrm{z}$ is $16: 1 / 16: 1 / 18: 1$ (or 16:1/18:1/16:1) TAG. While the mass spectrometer cannot distinguish between oleic acid (18:1 $\Delta 9)$ and cis-vaccenic acid (18:1 111$)$ isomers, which have identical masses, it is likely that the 18:1-containing TAG species reflect the high levels of $18: 1 \Delta 11$ produced by the engineered seeds. Other TAG species that are unique to high omega-7 transgenic lines and not found in wild-type camelina oil include $866.8 \mathrm{~m} / \mathrm{z}(16: 1 / 18: 3 / 18: 3), \mathrm{m} / \mathrm{z} 868.8$ (16:1/18:2/ $18: 3)$, and high amounts of $16: 1 / 18: 1 / 18: 3(870.8 \mathrm{~m} / \mathrm{z})$ and 16:1/18:1/18:1 (874.8 $\mathrm{m} / \mathrm{z})$.

\section{Seed weight, oil content and germination are unaffected by omega-7 unsaturated fatty acid accumulation}

The effect of high levels of omega-7 unsaturated fatty acids on seed total oil content, seed weight and seed germination was also examined (Table 4). Under greenhouse conditions, the oil content of seeds from nonengineered plants and seeds from high omega7 unsaturated fatty acid lines was $\sim 32 \%$ of seed weight. Similarly, the germination rate of both sets of seeds was $\sim 97 \%$ (Table 4). These results suggest that accumulation of omega- 7 unsaturated fatty acids to levels of $65 \%$ of the total fatty acids does not negatively affect seed oil content or germination.

\section{Omega-7 unsaturated fatty acid accumulation affects the thermotropic properties of camelina oil}

Differential scanning calorimetry of the extracted oils revealed multiple transitions during cooling and heating (Figure 6). During cooling, the onset of crystallization occurred at 

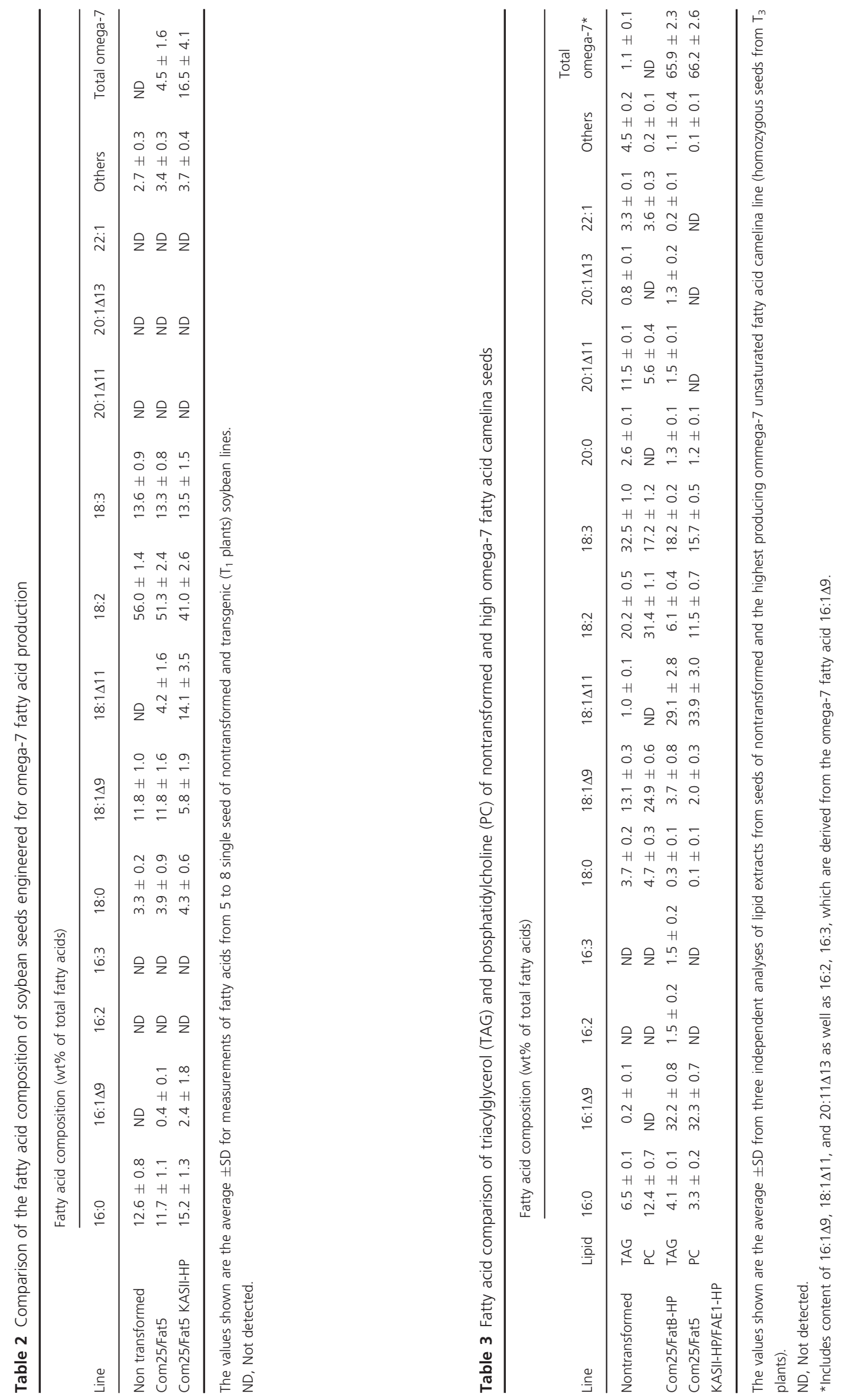


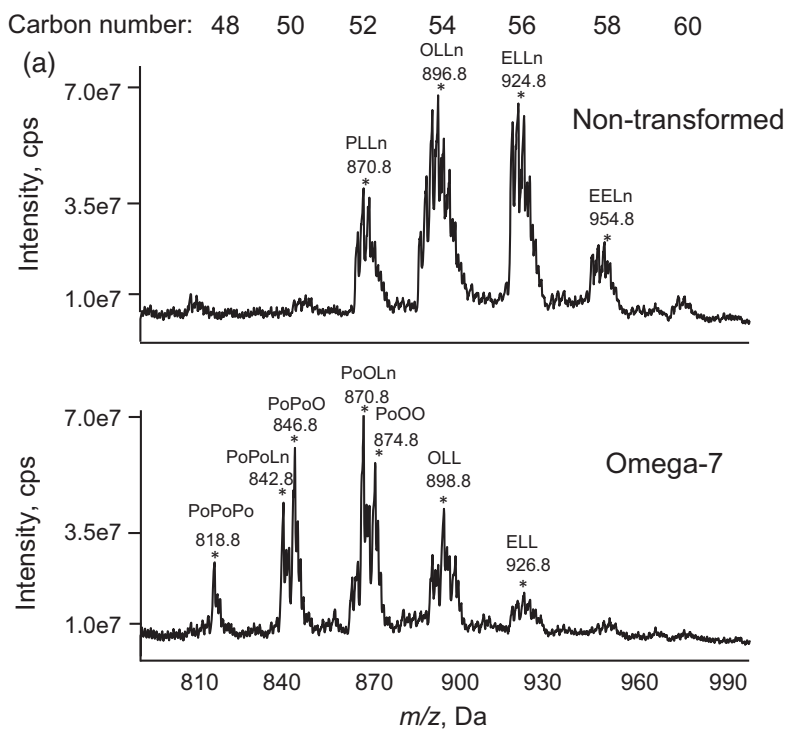

(b)

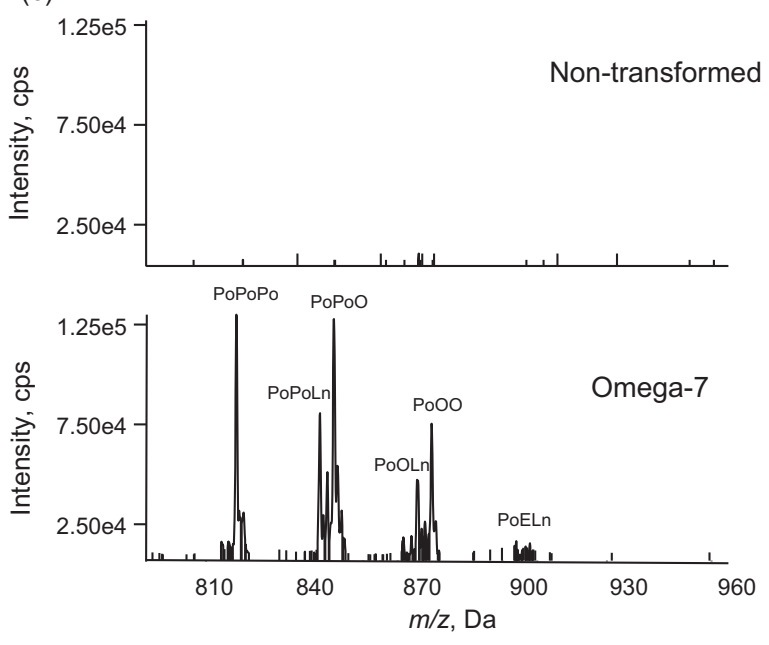

Figure 5 TAG profiling of oil extracts from seeds from nontransformed and high omega-7 lines by ESI-MS/MS. Q1 scan (a) comparing intact, ammoniated $\left(\mathrm{M}+\mathrm{NH}_{4}\right)$ TAG species present in wild-type and high omega-7 camelina oil. Signal intensity is expressed in counts per second (cps). Major TAG species are indicated with an asterisk (*). Peaks to the right of the asterisk represent TAG species with the same carbon number but fewer double bonds ( $\mathrm{m} / \mathrm{z}$ increasing by 2 mass units per double bond lost), and peaks to the left of the asterisk represent TAG species with the same carbon number but with more double bonds $(\mathrm{m} / \mathrm{z}$ decreasing by 2 mass units for each additional double bond). (b) Neutral loss scans for loss of 16:1 fatty acid ( $\mathrm{m} / \mathrm{z} 271)$ show that novel 16:1-containing TAG species PoPoPo $(m / z=818.8)$, PoPoLn $(m / z=842.8)$, PoPoO $(m / z=846.8)$, PoOLn $(m / z=870.8)$, PoOO $(m / z=874.8)$ are not found in seed oil from nontransformed lines but are abundant in oil from high omega-7 lines. The novel 16:1 containing TAGs were confirmed by product ion scans that show the presence of novel DAGs, PoPo $(\mathrm{m} / \mathrm{z}=547.8)$ and PoLn $(\mathrm{m} /$ $z=571.8$ ) (Figure $S 4$ and Figure S5). $P=16: 0, P o=16: 1, O=18: 1$, $L=18: 2, L n=18: 3, E=20: 1$.

$-6.0^{\circ} \mathrm{C}$ for oil from the nontransformed seeds, while crystallization did not begin until $-30.2{ }^{\circ} \mathrm{C}$ for oil from the transgenic line enriched in omega-7 fatty acids (Table 4 and Figure 6a). For both oils, a major crystallization exotherm was detected at
Table 4 Seed weight, oil content and germination rate of seeds from nontransformed plants and seeds from highest omega-7 unsaturated fatty acid accumulating line (transformed with Com25/FatB-

HP + Com25/Fat5/KASII-HP/FAE1-HP constructs; 'High Omega-7'). $T_{3}$ seeds from a homozygous engineered line were used for measurement of seed weight, seed oil content and germination rate

\begin{tabular}{llll}
\hline Line & Seed weight $(\mathrm{mg})^{*}$ & Oil content $(\%)^{\dagger}$ & Germination $(\%)^{*}$ \\
\hline Nontransformed & $0.95 \pm 0.05$ & $31.9 \pm 2.7$ & $96.3 \pm 1.5$ \\
High omega-7 & $0.95 \pm 0.04$ & $32.0 \pm 0.7$ & $95.7 \pm 2.5$ \\
\hline
\end{tabular}

*Values are the average seed weight \pm SD from measurements of 80 seeds from a nontransformed and the highest omega-7 unsaturated fatty acidproducing line $(n=3)$.

$\dagger$ Values are the average oil content \pm SD from analyses of three independent measurements.

*Values shown are the average germination rate \pm SD from three soilcontaining trays, each sowed with 50 seeds, from the highest omega-7 unsaturated fatty acid-producing line and nontransformed plants.

lower temperatures; the midpoint $T_{\mathrm{m}}$ of this transition was $-62.5^{\circ} \mathrm{C}$ for the nontransformed and $-55.5^{\circ} \mathrm{C}$ for the high omega-7 oil.

During heating of the oils, complex melting and recrystallization behaviour was detected (Figure 6b). Oil from the nontransformed seeds began to melt at $-54.4^{\circ} \mathrm{C}$; the small endotherm was followed by an exothermic recrystallization event that preceded the largest endotherm, which had a midpoint $T_{\mathrm{m}}$ at $-11.2{ }^{\circ} \mathrm{C}$. A small shoulder was apparent following the main endotherm, and melting was completed at $3.1^{\circ} \mathrm{C}$. Oil from the high omega-7 line did not begin to melt until $-35.4{ }^{\circ} \mathrm{C}$ and was characterized by two endotherms, with $T_{m}$ 's at $-25.5^{\circ} \mathrm{C}$ and $-15.3{ }^{\circ} \mathrm{C}$. In some samples, melting was complete at $-5.7^{\circ} \mathrm{C}$, while in other samples, a small shoulder appeared on the endotherm and the melt was not complete until $10^{\circ} \mathrm{C}$. This shoulder may indicate the presence of some impurity in the extracted oil or some heterogeneity among the samples.

In addition to the lowering of $T_{\text {on }}$ for crystallization of oil from the high omega-7 line, freezing was completed over a temperature range that was almost $20{ }^{\circ} \mathrm{C}$ less than for the oil from nontransformed seeds (Table 5). Similarly, melting transitions during heating occurred over a wider temperature for the oils from nontransformed seeds than for the omega-7 enriched oils (Table 5). In both samples, polymorphism in the TAG crystallization and melting processes also contributes to the complexity of the thermal signals. TAGs typically show multiple endothermic events during heating, as less stable crystalline forms melt, reorient themselves, recrystallize and then melt again, eventually completely liquefying (Tan and Che Man, 2002). Such polymorphism may explain the multiple events seen in heating scans of oils from nontransformed and high omega-7 camelina (Figure 6b).

\section{Discussion}

The results presented here demonstrate the ability to engineer the production of omega-7 unsaturated fatty acids to $\sim 60-65 \%$ of the total fatty acids of seeds of camelina, compared with $\sim 2 \%$ of the fatty acids of seeds from nontransformed plants. This large shift in fatty acid unsaturation from the typical C18 $\Delta 9$ unsatu- 

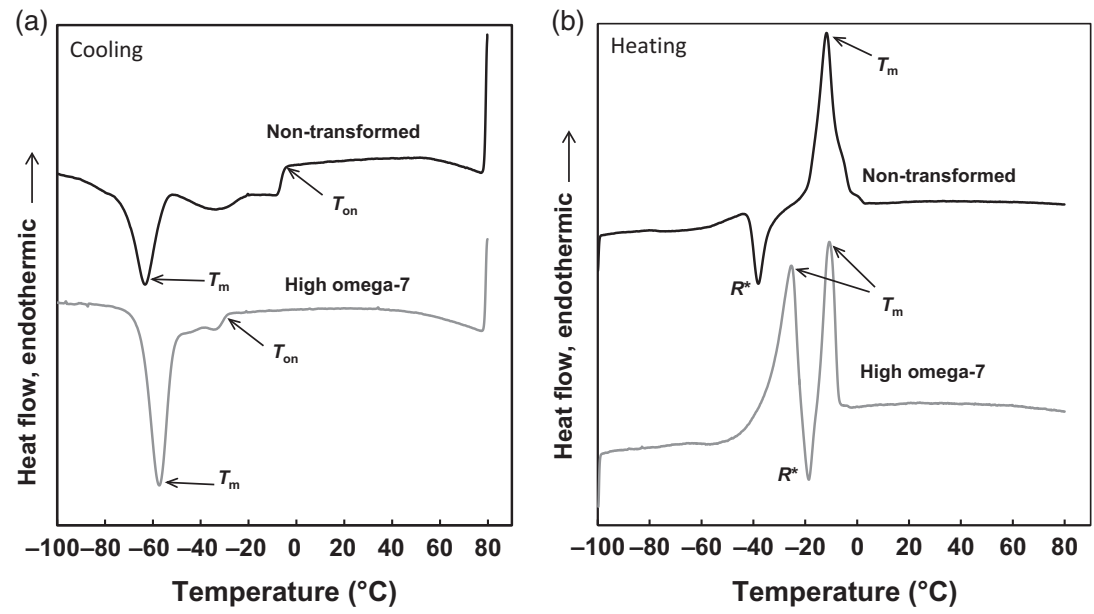

Figure 6 Differential scanning calorimetry (DSC) of TAG from nontransformed and high omega-7 camelina seeds. Representative cooling (a) and heating (b) scans show complex crystallization and melting behaviours of the lipids. Cooling scans (a) reveal a lower crystallization onset temperature, $T_{\text {on, }}$, for TAG enriched in omega-7 unsaturated fatty acids compared to TAGs from nontransformed seeds. Subsequent heating (b) shows initial melting endotherms, followed by recrystallization $\left(R^{*}\right)$ and subsequent melting for oils from both lines. $T_{m}$ indicates the midpoint transition temperature for crystallization exotherms (a) and melting endotherms (b).

Table 5 Thermal properties of TAGs extracted from seeds of nontransformed and transgenic camelina

\begin{tabular}{llllllll}
\hline & Crystallization & Crystallization & Melting onset \\
Line & $T_{\text {on }}\left({ }^{\circ} \mathrm{C}\right)$ & $T_{\mathrm{m}}\left({ }^{\circ} \mathrm{C}\right)$ & $\begin{array}{l}\text { Melting 1st } \\
\left.{ }^{\circ} \mathrm{C}\right)\end{array}$ & $\begin{array}{l}\text { Recrystallization } \\
\text { nadir }\left({ }^{\circ} \mathrm{C}\right)\end{array}$ & $\begin{array}{l}\text { Melting 2nd } \\
T_{\mathrm{m}}\left({ }^{\circ} \mathrm{C}\right)\end{array}$ & $\begin{array}{l}\text { Melting offset } \\
T\left({ }^{\circ} \mathrm{C}\right)\end{array}$ & $\begin{array}{l}\text { Melting } \\
\text { range }\left({ }^{\circ} \mathrm{C}\right)\end{array}$ \\
\hline Nontransformed & $-6.5 \pm 1.1$ & $-62.5 \pm 2.0$ & $-56.1 \pm 2.7$ & $-45.8 \pm 2.3$ & $-39.7 \pm 4.3$ & $-12.5 \pm 1.9$ & $3.1 \pm 1.2$ \\
High omega-7 & $-30.2^{*} \pm 4.0$ & $-55.5 \pm 2.5$ & $-35.4^{*} \pm 1.2$ & $-25.5^{*} \pm 0.6$ & $-17.7^{*} \pm 1.8$ & $-10.3 \pm 0.5$ & $3.6 \pm 7.8$ \\
\hline
\end{tabular}

Values represent average ( \pm SD) acquired from scans of three (Nontransformed) or four (High omega-7) independent TAG extractions. Values for high Omega-7 TAGs designated by * differ $(P<0.01)$ from those from TAG of nontransformed camelina.

rated fatty acid pathway did not result in significant reductions in total seed fatty acid content, seed weight or seed germination under greenhouse conditions. Although no empirical differences in growth of the high omega-7 lines relative to nontransformed plants, field studies are required to conclusively establish that this trait has no agronomic yield penalty.

Key to this engineering strategy is the use of a mutant $\Delta 9$-acyl$A C P$ desaturase with activity enhanced for 16:0-ACP, rather than 18:0-ACP and an ER $\triangle 9-16: 0-C O A$ desaturase. The seed-specific expression of these enzymes combined with KASIl suppression to enhance 16:0-ACP substrate pools resulted in seeds with $44 \%$ omega-7 unsaturated fatty acids. The inclusion of a second $\Delta 9$ 16:0-ACP desaturase transgene and RNAi suppression of FatB increased the total amount of omega-7 unsaturated fatty acids to $\sim 60-65 \%$ of the total fatty acids. Although it is not possible to dissect the relative contributions of these additional transgenes, we hypothesize that RNAi suppression of the FatB thioesterase (FatB-HP) provided further increases in 16:0-ACP pool sizes for the $\triangle 9-16: 0-A C P$ desaturase.

An unexpected outcome of the metabolic engineering strategy was a large reduction in total saturated fatty acids in our final transgene combination that included FatB-HP along with expression of 16:0-ACP and -CoA desaturases. In seeds from these lines, saturated fatty acids accounted for $\sim 5 \%$ of the total fatty acids compared with $\sim 12 \%$ in seeds from nontransformed seeds. Strikingly, not only 16:0 levels were reduced in seeds from these lines, but also $18: 0$ accounted for $\sim 0.3 \%$ of the total fatty acids in transgenic seeds compared with 3.5\% in nontransformed seeds. Notably, these saturated fatty acid levels in engineered seeds were lower than those recently reported in Arabidopsis seeds modified for expression of the Fat5 $\Delta 9-16: 0$ CoA desaturase (Fahy et al., 2013).
Another interesting observation from these studies was the near identical content of omega-7 unsaturated fatty acids in TAG and PC of engineered seeds. This observation indicates that camelina seeds do not exclude omega-7 fatty acids from PC, despite the fact that these fatty acids are likely not synthesized on this lipid, in contrast to C18 polyunsaturated fatty acids. Given that germination of engineered seeds is apparently not negatively affected, it is likely that seeds are able to tolerate high levels of omega-7 unsaturated fatty acids in membrane lipids.

The availability of our engineered camelina lines provides an opportunity to evaluate high omega-7 oils in industrial applications, such as 1-octene production, and in nutraceuticals. As a prelude to more extensive functional evaluation of high omega-7 unsaturated camelina oil, we conducted thermal analysis of seed oil from our top engineered line. As indicated by ESI-MS/MS analyses, TAG species from the engineered lines have distinct fatty acid compositions relative to TAG from seeds of nontransformed lines, including reductions in species enriched in C18 polyunsaturated fatty acids and a corresponding increase in species with monounsaturated fatty acids and C16 fatty acids. These differences in TAG composition are reflected in distinct thermotropic properties of the high omega-7 unsaturated camelina oil compared with oil from nontransgenic seeds. The high omega-7 unsaturated fatty acid oil has $\sim 18.5 \%$ more C 16 fatty acids and $\sim 10 \%$ less $\mathrm{C} 18$ fatty acids relative to oil from nonengineered seeds (Table 1). This overall decrease in fatty acid chain length should contribute to lower melting points among the TAGs of the high omega-7 line (Knothe and Dunn, 2009). This effect of decreased chain length counters the slight increase in melting points one might expect from the shift from the diunsaturated 18:2 fatty acids to the monounsaturated omega-7 fatty acids. In a recent study of the melting points of several TAG 
species, Knothe and Dunn (2009) reported that TAG of 16:1 $\Delta 9$ show two endothermic peaks during melting with $T_{m}$ values of around -28 and $-22{ }^{\circ} \mathrm{C}$. By comparison, the $T_{\mathrm{m}}$ of TAG containing $18: 1 \Delta 11$ was $1.0^{\circ} \mathrm{C}$ and $T_{\mathrm{m}}$ for TAGs of $18: 2 \Delta 912$ was $-12.7^{\circ} \mathrm{C}$ (Knothe and Dunn, 2009). Thus, the melting points reported here are consistent with TAG containing mixtures of these fatty acids. It is expected that these alterations in thermotropic properties of camelina oil may have important implications for the use of this oilseed in biodiesel applications. In addition, the broader temperature transition range for the oil from nontransformed seeds may indicate more varied TAG molecular species composition, which would cause less cooperativity during freezing and melting. It may also be indicative of the decreased saturated fatty acid content in the transgenic line, as oils with greater saturated fatty acid content melt over a broader temperature range than oils with more unsaturated fatty acids (Tan and Che Man, 2000).

The results reported here are among the highest levels of novel fatty acids generated in an engineered oilseed crop. We did observe that seed-specific expression of the $\triangle 9$ 16:0-ACP and COA desaturases with KASII suppression in soybean yielded less than half the omega-7 unsaturated fatty acids compared to camelina engineered with the same transgene combination. Although we do not know the basis for this difference, this observation indicates that our engineering strategy may need additional optimization for transfer to other oilseeds. Future studies for the optimization of this trait may include introduction of additional transgenes for key enzymes, including the Com25 or FAT5 desaturases, and more detailed quantification of target protein levels to determine whether maximal metabolic capacity for omega-7 fatty acid production has been achieved in our current top-performing lines. In addition, we hypothesize that additional increases in omega-7 unsaturated fatty acids can be achieved by seed-specific suppression of the native camelina $\triangle 9-18: 0-A C P$ desaturase to more completely shift flux to $\triangle 9-16: 0-A C P$ desaturation, although the impact of this modification on agronomic fitness, including seed germination, is difficult to predict.

\section{Experimental procedures}

\section{Vector construction}

Clones encoding KASII were identified from the published camelina seed transcriptome (Nguyen et al., 2013). To construct the camelina KASII sense arm, a 162 base pair region of camelina KASII was amplified with the oligonucleotide primers: $5^{\prime}$ GGGCTGCAGAAACAGCAACTATCTACGCA-3' and 5'- GGGCT CGAGGCGGCCGCATGTAATTTATCTGTT-3' (added restriction sites are italicized). The Pstl/Xhol restriction enzyme fragment was used to replace the KASII sense arm in a modified version of a previously described plasmid utilizing a FAD2 intron in the previously described vector pGEMT-KASIIHP-FAD2HP (Okuley et al., 1994; Pidkowich et al., 2007). The KASII antisense sequence used was PCR amplified from camelina developing seed CDNA using oligonucleotide primers with flanking EcoRI/Notl and Nhel restriction sites: 5'- GGGCTAGCAGCAACTATCT ACGCA-3' and 5'- GGGGAATTCGCGGCCGCATGTAATTTATCT GTT-3'. The ECoRI/Nhel restriction enzyme fragment was used to replace the Arabidopsis KASII antisense arm in pGEMT-KASIIHPFAD2HP. The KASII sense, antisense and FAD2 intron were assembled and cloned into plasmid pBCon123-Hyg (Zhang et al., 2013) under the control of the soybean $\alpha$ 'subunit of $\beta$-conglycinin promoter and phaseolin $3^{\prime}$ UTR. A cassette including the soybean glycinin-1 promoter and $3^{\prime} U T R$, KASIl sense, KASIl antisense and FAD2 intron was excised using Hindlll and cloned into the binary vector pBinGlyRed2 (Nguyen et al., 2013) to generate pBinGlyRed-CsKasll-HP.

A codon-optimized Fat5 gene from C. elegans (Fahy et al., 2013; Watts and Browse, 2000) was synthesized (GenScript USA Inc., Piscataway, NJ) with the addition of flanking Notl restriction sites. The Notl fragment was cloned into the vector pKMS3 (Nguyen et al., 2013) to place Fat5 under the control of the soybean glycinin-1 promoter and 3'UTR to create pKMS3-Fat5S. A cassette comprising the glycinin-1 promoter and $3^{\prime} U T R$ flanking Fat5 gene was transferred into to pBinGlyRed-CsKasll to make pBinGlyRed-CsKasll-HP/Fat5. A codon-optimized Com25 open reading frame was synthesized (GenScript USA Inc.) to include flanking ECoRl and Xhol at the $5^{\prime}$ and $3^{\prime}$ ends, respectively. Com25 was digested with ECoRI and Xhol and cloned into the corresponding sites of pBinGlyRed-CsKasll-HP/Fat5 under control of the soybean glycinin-1 promoter to generate pBinGlyRedCsKasll-HP/Fat5/Com25.

A previously described FAE1 RNAi suppression cassette regulated by a glycinin-1 promoter (Nguyen et al., 2013) was ligated as an Ascl restriction enzyme fragment and cloned into the Mlul restriction site of pBinGlyRed-CsKasll-HP/Fat5/com25 to obtain the final vector pBinGlyRed-CsKasll-HP/Fat5/Com25/FAE1-HP (short name is pBinGlyRed/C25/F5/K2/F1, Figure 2, top).

A Com 25 fragment flanked by EcoRl/Xhol restriction sites was cloned into the corresponding sites of pBinGlyRed2 (Nguyen et al., 2013) to obtain the vector pBinGlyRed-Com25. For Fat5, the cassette containing the glycinin-1 promoter, Fat5 open reading frame and the glycinin-1 3'UTR was restricted from vector pKMS3-Fat5S using Ascl and cloned into the corresponding site in pBinGlyRed-Com25 to create pBinGlyRed-Com25/Fat5.

A camelina FatB RNAi suppression cassette was constructed similarly to the KASII hairpin structure above with two pairs of primers 5'-GGGCTGCAGAAAC AATGTAAAGTACATTGGGT3'and 5'- GGGCTCGAGGCGGCCGCACTCCACTCTGTTCTTCCTC -3'; 5'- GGGCTAGC AATGTAAAGTACATTGGGT -3' and 5'GGGGAATTCGCGGCCGCACTCCACTCTGTTCTTCCTC - $3^{\prime}$. The FatB sequence was obtained from the published camelina seed transcriptome (Nguyen et al., 2013), and CDNA generated from developing camelina seeds was used for the PCR template. Com25 open reading frame-containing cassette was cloned in to vector pBinGlyBar1 (Nguyen et al., 2013) to obtain pBinGlyBar1/ Com25/FatB-HP (Figure 2, bottom). Both transgenes in this vector were placed under the control of the seed-specific soybean glycinin-1 promoter.

\section{Camelina transformation}

Vectors pBinGlyRed-CsKasll-HP, pBinGlyRed-Com25, pBinGlyRedCom25/Fat5, pBinGlyRed-CsKasll-HP/Fat5, pBinGlyRed-CsKasllHP/Fat5/Com25, pBinGlyRed-CsKasll-HP/Fat5/Com25/FAE1-HP were transformed into Camelina sativa cv. Sunesson using the previously described floral vacuum infiltration method (Lu and Kang, 2008) and transformed seeds were selected by DsRed fluorescence as previously described (Nguyen et al., 2013). The highest omega-7 unsaturated fatty acid-producing line transgenic line pBinGlyRed-CsKasll-HP/Fat5/Com25/FAE1-HP (homozygous $T_{3}$ lines) was retransformed with pBinGlyBar1/Com25/ FatB-HP (Figure S1), and transformed plants were selected by Basta (glufosinate) resistance as described previously (Nguyen et al., 2013). Camelina plants were grown under greenhouse conditions with $14 \mathrm{~h}$ day length $\left(24-26^{\circ} \mathrm{C}\right)$ and $8 \mathrm{~h}$ dark 
$\left(18-20^{\circ} \mathrm{C}\right)$ with natural and supplemental lighting at 400 $500 \mu \mathrm{moles} / \mathrm{m}^{2} / \mathrm{s}$ as described previously (Nguyen et al., 2013).

\section{Preparation of soybean transformation construct and transformation}

Vector pBinGlyBar1-Com25/Fat5 is similar with vector pBinGlyRed-Com25/Fat5 but clone in to pBinBar1 backbone (Nguyen et al., 2013). Com25 fragment was cloned into pBinGlyBar1 vector at EcoRl/Xhol, and Fat5 cassette including soybean glycinin-1 promoter and 3'UTR from pKMS3-Fat5S was cloned at Ascl.

The soybean KASII RNAi cassette was assembled similar camelina KASII RNAi. The KASII sense was amplified with the oligonucleotide primers: 5'- GGGCTGCAGAAACAAC AGATTTCAGAGCAC-3' and 5'- GGGCTCGAGGCGGCCGCCTATC AACATTCTGAT-3'. The soybean KASII antisense was amplified with oligonucleotide primers 5'- GGGCTAGCAGCAACAGA TTTCAGAGCAC-3' and 5'- GGGGAATTCGCGGCCGCCTATCAA CATTCTGAT-3' The soybean KASII RNAi cassette was added to pBinGlyBar1-Com25/Fat5 at Mlul to create pBinGlyBar1-Com25/ Fat5/KASII.

The soybean cultivar Thorne was transformed through an Agrobacterium-mediated transformation system as described (Zhang et al., 1999). The final two binary vectors pBinGlyBar1C25F5 and pBinGlyBar1-C25F5K2 carrying a Basta resistance gene as a selection marker and targeted genes (Com25, Fat5, KASII hairpin) were delivered into the $A$. tumefaciens strain NTL4 by tri-parental mating and were used for transformation into soybean (Zhang et al., 1999). Primary transformants were screened by the leaf paint assay for herbicide tolerance (Zhang et al., 1999). Transgenic soybean plants were grown in a greenhouse with a $14-\mathrm{h}$ photoperiod and $28{ }^{\circ} \mathrm{C} / 26^{\circ} \mathrm{C}$ day/night temperature.

\section{Gas chromatographic analysis of fatty acid compositions}

Fatty acid methyl esters (FAMEs) were prepared from camelina seeds by transesterification with trimethylsulfonium hydroxide (Butte, 1983) or with $2.5 \%$ (v/v) sulphuric acid/methanol as previously described (Nguyen et al., 2013). Fatty acid methyl esters were analysed by gas chromatography with flame ionization detection as previously described (Nguyen et al., 2013). Double bond positions in omega-7 monounsaturated fatty acid $16: 1 \Delta 9,18: 1 \Delta 11$ and 20:1 $\Delta 13$ (Figure S1) from engineered were determined GC-MS analysis of dimethyl disulphide adducts prepared from fatty acid methyl esters as described (Yamamoto et al., 1991).

\section{Analysis of triacylglycerol and phosphatidylcholine fatty} acid compositions of camelina seeds

Lipids were extracted from camelina seeds $(25-30 \mathrm{mg}$ ) by grinding to a fine powder in $3 \mathrm{~mL}$ methanol/chloroform (2: 1 v/v). Samples were incubated for 30-60 min at room temperature with agitation, and then, $1 \mathrm{~mL}$ chloroform and $1.8 \mathrm{~mL}$ water were added (Bligh and Dyer, 1959). The tubes were shaken well, and aqueous and organic phases were separated by centrifugation. The organic phase was transferred into a new tube, and the solvent evaporated under $\mathrm{N}_{2}$. Lipids were redissolved in $1 \mathrm{~mL}$ heptane and applied to an equilibrated $3 \mathrm{~mL}$ Supelclean LC-Si SPE column (Supelco, Saint Louis, MO). The TAG fraction was eluted with $4 \mathrm{~mL}$ of $80: 20(\mathrm{v} / \mathrm{v})$ heptane/ethyl ether. The column was then washed with $4 \mathrm{~mL}$ acetone. Total phospholipids were subsequently eluted with $5 \mathrm{~mL}$ of methanol:chloroform:water
(100: $50: 40 \mathrm{v} / \mathrm{v} / \mathrm{v}$ ), and $1.7 \mathrm{~mL}$ of chloroform and $1.7 \mathrm{~mL}$ of water were added to the eluent. After shaking and centrifugation, the total phospholipids in the organic layer were recovered. The phospholipid fraction was then resolved on silica TLC in chloroform/methanol/water/ammonium hydroxide (65/35/3/2.5 $\mathrm{v} / \mathrm{v} / \mathrm{v} / \mathrm{v})$, and the $\mathrm{PC}$ band was scraped from plate and directly transesterified in $2.5 \%$ sulphuric acid/methanol ( $v / v)$ as described previously (Nguyen et al., 2013). An aliquot of the TAG fraction was also transesterified in $2.5 \%$ sulphuric acid/methanol (v/v) and $250 \mu \mathrm{L}$ of toluene (Nguyen et al., 2013). Following heating at $95{ }^{\circ} \mathrm{C}$ for $45 \mathrm{~min}$, fatty acid methyl esters were recovered and analysed by gas chromatography-flame ionization detection as described (Cahoon et al., 2006).

\section{ESI-MS/MS analysis of TAG molecular species}

Mass spectrometry analyses were conducted using an Applied Biosystems (Foster City, CA) 4000 QTRAP linear ion trap quadrupole mass spectrometer to characterize TAG molecular species. The total neutral lipid extract for ESI-MS/MS analysis was prepared as described for seed oil content measurement below but without added internal standard and diluted 1:5000 in water/ isopropyl alcohol/methanol (55: $35: 10 \mathrm{v} / \mathrm{v} / \mathrm{v})$ containing $25 \mathrm{~mm}$ ammonium formate and $4 \mu \mathrm{L} / \mathrm{L}$ formic acid and directly infused into the mass spectrometer at a rate of $20 \mu \mathrm{L}$ per minute. A Q1 scan (positive mode) was performed over a mass range of 450 $1200 \mathrm{~m} / \mathrm{z}$ with a cycle time of $2 \mathrm{~s}$. Data were collected for 150 cycles. Instrument settings were as follows: Source temperature $300{ }^{\circ} \mathrm{C}$, ESI needle voltage $5.5 \mathrm{kV}$ (positive mode), desolvation potential (DP) 90, entrance potential (EP) 10, Curtain gas (CUR) 10, and gas 1 (GS1) 50 arbitrary units, gas 2 (GS2) 40 arbitrary units. Product ion spectra were generated for selected TAG molecular species using the instrument settings and flow rates described above, and applying collision energy of 54 and a collision exit potential of 11 . Scans were taken over a mass range of $200-1000 \mathrm{~m} / \mathrm{z}$ with a cycle time of $2 \mathrm{~s}$, and data files generated with 50-150 scans. Neutral loss spectra showing the loss of a specific fatty acid from TAG species were generated by direct infusion using instrument settings described above and a source temperature of $400{ }^{\circ} \mathrm{C}$. Scans were taken over a mass range of $500-1475 \mathrm{~m} / \mathrm{z}$ with a cycle time of $3 \mathrm{~s}$. Data was collected for five cycles. Neutral loss of the following fatty acids was monitored: C8:0 $(161.0 \mathrm{~m} / \mathrm{z})$, C10:0 $(189.1 \mathrm{~m} / \mathrm{z}), C 12: 0$ $(217.1 \mathrm{~m} / \mathrm{z}), \quad C 14: 0(245.1 \mathrm{~m} / \mathrm{z}), \quad \mathrm{C} 16: 0(273.1 \mathrm{~m} / \mathrm{z}), \quad \mathrm{C} 16: 1$ $(271.1 \mathrm{~m} / \mathrm{z}), \quad C 18: 0 \quad(301.1 \mathrm{~m} / \mathrm{z}), \quad C 18: 1 \quad(299.1 \mathrm{~m} / \mathrm{z}), \quad C 18: 2$ $(297.1 \mathrm{~m} / \mathrm{z}), \quad C 18: 3 \quad(295.1 \mathrm{~m} / \mathrm{z}), \quad$ C20:0 $(329.1 \mathrm{~m} / \mathrm{z}), \quad C 20: 1$ $(327.1 \mathrm{~m} / \mathrm{z}), \quad C 22: 0 \quad(357.1 \mathrm{~m} / \mathrm{z}), \quad C 22: 1 \quad(355.2 \mathrm{~m} / \mathrm{z}), \quad C 24: 0$ $(385.2 \mathrm{~m} / \mathrm{z}), C 24: 1(383.2 \mathrm{~m} / \mathrm{z}), \mathrm{C} 26: 0(413.2 \mathrm{~m} / \mathrm{z})$ and $\mathrm{C} 26: 1$ (411.2 m/z).

\section{Seed oil content measurement}

Total lipids were extracted from $30 \mathrm{mg}$ of transgenic camelina seeds as described above with the inclusion of triheptadecanoin (NuChek Prep, Elysian, MN) internal standard, and total fatty acids were measured by transesterification and subsequent analysis by gas chromatography/flame ionization detection as previously described (Nguyen et al., 2013). Fatty acids were quantified relative to the gas chromatographic response of heptadecanoyl methyl ester from the internal standard.

\section{Seed germination measurement}

Three soil-containing trays, each sowed with 50 seeds, for the high omega-7 unsaturated line and a nontransformed line were 
maintained under greenhouse conditions. After 4 days, germinated seeds from each line were counted.

\section{Differential scanning calorimetry of camelina seed oil}

Camelina seeds $(500 \mathrm{mg}$ ) were ground to a fine powder with glass stirring rod in $2 \mathrm{~mL}$ of heptane and then incubated at $40{ }^{\circ} \mathrm{C}$ for $1 \mathrm{~h}$. The oil extract in heptane was washed with $1 \mathrm{~mL}$ of water and transferred to a $100 \times 30 \mathrm{~mm}$ class screw cap tube. The oil fraction was dried under nitrogen and heated to $55^{\circ} \mathrm{C}$ in a dry-block heater to assure that it was liquid prior to transfer via syringe into preweighed aluminium volatile sample pans $(20 \mu \mathrm{L}$ volume). The pans were hermetically sealed and weighed to obtain the sample weights, which ranged from 2 to $8 \mathrm{mg}$. Calorimetry was carried out using a DSC-7 (Perkin Elmer, Norwalk, CT) with liquid $\mathrm{N}_{2}$ cooling and a helium purge gas. The method used was modified from AOCS standard protocol $\mathrm{Cj}$ 1-94, which recommends holding samples at $80^{\circ} \mathrm{C}$ for $10 \mathrm{~min}$, scanning at $10{ }^{\circ} \mathrm{C} / \mathrm{min}$ to $-40{ }^{\circ} \mathrm{C}$, holding for $30 \mathrm{~min}$ at $-40{ }^{\circ} \mathrm{C}$, then scanning at $5{ }^{\circ} \mathrm{C} / \mathrm{min}$ to $80^{\circ} \mathrm{C}$ (AOCS, 2004; Ribiero et al., 2009). In our experiments, samples were cooled to -80 or $-100{ }^{\circ} \mathrm{C}$ to enable detection of the crystallization exotherms. The calorimeter was calibrated to the onset melting temperatures of indium $\left(T_{\mathrm{m}}=156.6^{\circ} \mathrm{C}\right)$ and heptane $\left(T_{\mathrm{m}}=-90.56^{\circ} \mathrm{C}\right)$, heated at $5{ }^{\circ} \mathrm{C} / \mathrm{min}$. Indium was used to calibrate the melting enthalpy $(\Delta \mathrm{H}=28.45 \mathrm{~J} / \mathrm{g})$ at $5^{\circ} \mathrm{C} / \mathrm{min}$. Indium was used to calibrate the melting enthalpy $(\Delta \mathrm{H}=28.45 \mathrm{~J} / \mathrm{g})$ at $5^{\circ} \mathrm{C} / \mathrm{min}$. Because the cooling and heating rates differed, and the DSC was calibrated to the heating rate, the melting and crystallization onset temperature of indium was measured to enable comparison of temperatures obtained from cooling and heating curves. The crystallization onset of indium was measured at $154.3^{\circ} \mathrm{C}$ while cooling at $10{ }^{\circ} \mathrm{C}$. Data obtained from the cooling and heating scans were analysed using the software provided by Perkin-Elmer for the Model 1020 Controller. $T_{\mathrm{m}}$ represents the temperature of the peak maximum or minimum for endotherms and exotherms, respectively. $T_{\text {on }}$ represents the onset temperature for crystallization during cooling (Tan and Che Man, 2000).

\section{Acknowledgements}

The project was supported by funding from the US Department of Agriculture-Agriculture and Food Research Initiative 200905988 and the Nebraska Soybean Board to TEC and EBC and support from the US DOE BES program to JS. We thank Manuel Rodriguez Rodriguez for expert technical assistance with oil thermal analysis.

\section{References}

AOCS (2004) AOCS Official Method Cj 1-94. Official Methods and Recommended Practices of the American Oil Chemists' Society, 4th edn. Champaign, IL: American Oil Chemists' Society

Bligh, E.G. and Dyer, W.J. (1959) A rapid method for total lipid extraction and purification. Can. J. Biochem. Physiol. 37, 911-917.

Bridge, R.E. and Hilditch, T.P. (1950) 492. The seed fat of Macadamia ternifolia. J. Chem. Soc. 1950, 2396-2399.

Butte, W.J. (1983) Rapid method for the determination of fatty acid profiles from fats and oils using trimethyl-sulphonium hydroxide for transesterification. J. Chrom. 261, 142-145.

Cahoon, E.B. and Shanklin, J. (2000) Substrate-dependent mutant complementation to select fatty acid desaturase variants for metabolic engineering of plant seed oils. Proc. Nat. Acad. Sci. USA, 97, 1235012355 .
Cahoon, E.B., Dietrich, C.R., Meyer, K., Damude, H.G., Dyer, J.M. and Kinney, A.J. (2006) Conjugated fatty acids accumulate to high levels in phospholipids of metabolically engineered soybean and Arabidopsis seeds. Phytochemistry, 67, 1166-1176.

del Cardayre, S.B. (2013) Metathesis transformations of microbially-produced fatty acids and fatty acid derivatives. US Provisional Patent Application US 20130274529 A1

Chisholm, M.J. and Hopkins, C.Y. (1965) Fatty acids of Doxantha seed oil. J. Am. Oil Chem. Soc. 42, 49-50.

Ciubota-Rosie, C., Ruiz, J.R., Ramos, M.J. and Pérez, Á. (2013) Biodiesel from Camelina sativa: a comprehensive characterisation. Fuel, 105, 572-577.

Curb, J.D., Wergowske, G., Dobbs, J.C., Abbott, R.D. and Huang, B. (2000) Serum lipid effects of a high-monounsaturated fat diet based on macadamia nuts. Arch. Intern. Med. 160, 1154-1158.

Durrett, T.P., Benning, C. and Ohlrogge, J. (2008) Plant triacylglycerols as feedstocks for the production of biofuels. Plant J. 54, 593-607.

Fahy, D., Scheer, B., Wallis, J.G. and Browse, J. (2013) Reducing saturated fatty acids in Arabidopsis seeds by expression of a Caenorhabditis elegans 16:0-specific desaturase. Plant Biotechnol. J. 11, 480-489.

Horn, P.J., Silva, J.E., Anderson, D., Fuchs, J., Borisjuk, L., Nazarenus, T.J., Shulaev, V., Cahoon, E.B. and Chapman, K.D. (2013) Imaging heterogeneity of membrane and storage lipids in transgenic Camelina sativa seeds with altered fatty acid profiles. Plant J. 76, 138-150

Kallio, H., Yang, B., Peippo, P., Tahvonen, R. and Pan, R. (2002) Triacylglycerols, glycerophospholipids, tocopherols, and tocotrienols in berries and seeds of two subspecies (ssp. sinensis and mongolica) of sea buckthorn (Hippophae rhamnoides). J. Agric. Food Chem. 50, 3004-3009.

Knothe, G.H. and Dunn, R.O. (2009) A comprehensive evaluation of the melting points of fatty acids and esters determined by differential scanning calorimetry. J. Am. Oil Chem. Soc. 86, 843-856.

Knothe, G., Van Gerpen, J.H. and Krahl, J. (2005) The Biodiesel Handbook. Champaign, IL: AOCS Press.

Lu, C. and Kang, J. (2008) Generation of transgenic plants of a potential oilseed crop Camelina sativa by Agrobacterium-mediated transformation. Plant Cell Rep. 27, 273-278.

Meier, M.A.R. (2009) Metathesis with oleochemicals: new approaches for the utilization of plant oils as renewable resources in plant science. Macromol. Chem. Phys. 210, 1073-1079.

Mozaffarian, D., Cao, H., King, I.B., Lemaitre, R.N., Song, X., Siscovick, D.S. and Hotamisligil, G.S. (2010) Trans-palmitoleic acid, metabolic risk factors, and new-onset diabetes in U.S. adults: a cohort study. Ann. Intern. Med. 153, 790-799.

Nguyen, H.T., Mishra, G., Whittle, E., Pidkowich, M.S., Bevan, S.A., Merlo, A.O., Walsh, T.A. and Shanklin, J. (2010) Metabolic engineering of seeds can achieve levels of omega-7 fatty acids comparable with the highest levels found in natural plant sources. Plant Physiol. 154, 18971904.

Nguyen, H.T., Silva, J.E., Podicheti, R., Macrander, J., Yang, W., Nazarenus, T.J., Nam, J.W., Jaworski, J.G., Lu, C., Scheffler, B.E., Mockaitis, K. and Cahoon, E.B. (2013) Camelina seed transcriptome: a tool for meal and oil improvement and translational research. Plant Biotechnol. J. 11, 759-769.

Okuley, J., Lightner, J., Feldmann, K., Yadav, N., Lark, E. and Browse, J. (1994) Arabidopsis FAD2 gene encodes the enzyme that is essential for polyunsaturated lipid synthesis. Plant Cell, 6, 147-158.

Pidkowich, M.S., Nguyen, H.T., Heilmann, I., Ischebeck, T. and Shanklin, J. (2007) Modulating seed $\beta$-ketoacyl-acyl carrier protein synthase II level converts the composition of a temperate seed oil to that of a palm-like tropical oil. Proc. Natl Acad. Sci. USA, 104, 4742-4747.

Ribiero, A.P.B., Basso, R.C., Grimaldo, R., Gioielli, L.A. and Gon alves, L.A.G. (2009) Instrumental methods for the evaluation of interesterified fats. Food Anal. Methods, 2, 282-302.

Rybak, A., Fokou, P.A. and Meier, M.A.R. (2008) Metathesis as a versatile tool in oleochemsitry. Eur. J. Lipid Sci. Technol. 110, 797-804.

Saleeb, W.F., Yermanos, D.M., Huszar, C.K., Storey, W.B. and Labanauskas, C.K. (1973) The oil and protein in nuts of Macadamia tetraphylla L. Johnson, Macadamia integrifolia Maiden and Betche, and their F1hybrid. J. Am. Soc. Hortic. Sci. 98, 453-456. 
Tan, C.P. and Che Man, Y.B. (2000) Differential scanning calorimetric analysis of edible oils: comparison of thermal properties and chemical composition. J. Am. Oil Chem. Soc. 77, 143-155.

Tan, C.P. and Che Man, Y.B. (2002) Recent development in differential scanning calorimetry for assessing oxidative deterioration of vegetable oils Trends Food Sci. Technol. 13, 312-318.

Watts, J.L. and Browse, J. (2000) A palmitoyl-CoA-specific $\Delta 9$ fatty acid desaturase from Caenorhabditis elegans. Biochem. Biophys. Res. Commun 272, 263-269.

Yamamoto, K., Shibahara, A., Nakayama, T. and Kajimoto, G. (1991) Determination of double-bond positions in methylene-interrupted dienoic fatty acids by GC-MS as their dimethyl disulfide adducts. Chem. Phys. Lipids 60, 39-50.

Yang, Z.H., Miyahara, H. and Hatanaka, A. (2011) Chronic administration of palmitoleic acid reduces insulin resistance and hepatic lipid accumulation in KK-Ay Mice with genetic type 2 diabetes. Lipids Health Dis. 10, 120

Zhang, Z., Xing, A., Staswick, P. and Clemente, T.E. (1999) The use of glufosinate as a selective agent in Agrobacterium-mediated transformation of soybean. Plant Cell Tissue Organ Cult. 56, 37-46.

Zhang, C., Cahoon, R.E., Hunter, S.C., Chen, M., Han, J. and Cahoon, E.B. (2013) Genetic and biochemical basis for alternative routes of tocotrienol biosynthesis for enhanced vitamin E antioxidant production. Plant J. 73, 628-639.

\section{Supporting information}

Additional Supporting information may be found in the online version of this article:

Figure S1 Structures of omega-7 fatty acids.

Figure S2 Scatter plots of omega-7 fatty acid content in transgenic single seed from highest producing line of camelina or soybean.

Figure S3 Mass spectra of thiomethyl adducts of fatty acid methyl esters of omega-7 monounsaturated fatty acids 16:1 $\Delta 9$, $18: 1 \Delta 11$, and $20: 1 \Delta 13$ from camelina seeds of a high omega- 7 camelina line.

Figure S4 Product ion scans of novel 16:1-containing TAG found in high omega-7 camelina oil.

Figure S5 DAG fragments resulting from product ion scans of $\mathrm{m} / \mathrm{z}$ $846.8(\mathrm{a}, \mathrm{b})$ and $\mathrm{m} / \mathrm{z} 870.8$ to confirm the fatty acid composition of the parent TAG species as PPLn in seed oil from nontransformed and PoPoO in high omega-7 fatty acid camelina oil. 